\title{
Active Earthing system for MV networks
}

\author{
F.J. Pazos ${ }^{1}$, A. Amezua ${ }^{2}$, I. Gutierrez ${ }^{2}$, J.M.Garcia ${ }^{3}$, R.Toledo ${ }^{3}$, G. Buigues ${ }^{4}$ and V. Valverde ${ }^{4}$ \\ ${ }^{1}$ Iberdrola Distribución Eléctrica, S.A.U. \\ Avenida San Adrián, 48, 48003 Bilbao (Spain) \\ e-mail: fj.pazos@iberdrola.es \\ ${ }^{3}$ Ingeteam Power Technology, S.A \\ Parque Tecnológico de Bizkaia, Edificio 110 \\ 48170 Zamudio (Spain) \\ e-mail: juanmari.garcia@ingeteam.com \\ e-mail: rafael.toledo@ingeteam.com \\ ${ }^{2}$ Ormazabal \\ Barrio Basauntz, 2 - 48140 Igorre (Spain) \\ e-mail: ama@ormazabal.com \\ e-mail: igu@ormazabal.com \\ ${ }^{4}$ Faculty of Engineering of Bilbao, UPV/EHU \\ Alameda de Urquijo s/n, 48013 Bilbao (Spain) \\ e-mail: garikoitz.buigues@ehu.es \\ e-mail: victor.valverde@ehu.es
}

\begin{abstract}
.
This paper describes and a new technology, the active earthing system, based on a multi-frequency power converter, which combines a new power electronic device with a protection and control system.

This system allows network enhanced operation and maintenance, overcoming some of the limitations of the traditional earthing systems, giving rise to relevant improvement in supply continuity.

The key questions and results of the validation along with lab testing data and field details are shown in this paper. The experiences of the first real application in a MV distribution substation being conducted in Gernika- Spain will be presented in ICREPQ'13 conference.
\end{abstract}

\section{Key words}

Active earthing, predictive maintenance, fault extinction, fault location.

\section{Introduction}

The type of neutral treatment has an important influence in the behaviour of Medium Voltage (MV) networks. It particularly affects voltage dips, swells and interruptions and also fault location, since earthing system determines several aspects of earth-faults such as fault current, duration and, to a certain extent, their probability of occurrence.

So far, traditionally the MV neutral has been earthed by means of passive elements, such as feeders' capacitances and/or discrete elements namely reactors and resistors or, simply, solidly earthed. In any case, when a fault happens, conventional earthing systems are not able to modify the current that flows through the fault or network neutral. It does not provide any other function while there is no fault in the network.
The active earthing system has the traditional advantages of a resonant network, such as arc suppression or low step and touch voltages. However, additional features are achieved by the injection of a controlled current in magnitude, phase and frequency. More details can be found in [1].

Two main tasks, described below, were considered for the system validation.

In the laboratory, a scale model of the active earthing system has been used connected to a MV network simulator. Earth faults have been simulated on different network configurations simulating several combinations of overhead line and cable sections, and a wide range of fault impedances.

In the field, real tests are being held. First of all, and prior to carry on fault extinction tests, the network is checked in order to make sure that isolation level and network component rating are able to withstand the new operating conditions, which are similar to those necessary for resonant networks. The active earthing system is used to create controlled phase-to-earth overvoltages in each phase and facilitate the process. Secondly, fault detection and location is tested for provoked (intentional) faults, including those of high impedance. Finally, the active earthing system performance is tracked and recorded for standalone operation.

\section{The "Active Earthing" concept}

"Active Earthing" means that any zero-sequence voltage can be created injecting the appropriate current, as states in (1), when desired and with the desired angle and frequency. 


$$
\overline{V_{N}}=\overline{I_{N}} \cdot \overline{Z_{N}}=3 \cdot \overline{V_{0}}=\overline{I_{0}} \cdot 3 \cdot \overline{Z_{0}}
$$

Therefore, phase-to-earth voltages can be modified on demand, as can be seen in equation (2):

$$
\overline{V_{\text {phase-to-earth }}}=\overline{V_{\text {phase-to-neutral }}}+\overline{V_{N}}
$$

- In normal operation conditions, with no fault in the network, neutral voltage can be modified, thus reducing or increasing one phase-to-earth voltage to any value, as shown in figure 1.

- During an earth-fault phase-to-earth voltages can be modified as well, being able to eliminate the voltage in the affected phase.

- Different frequencies can be injected in order to improve the protection system behaviour.

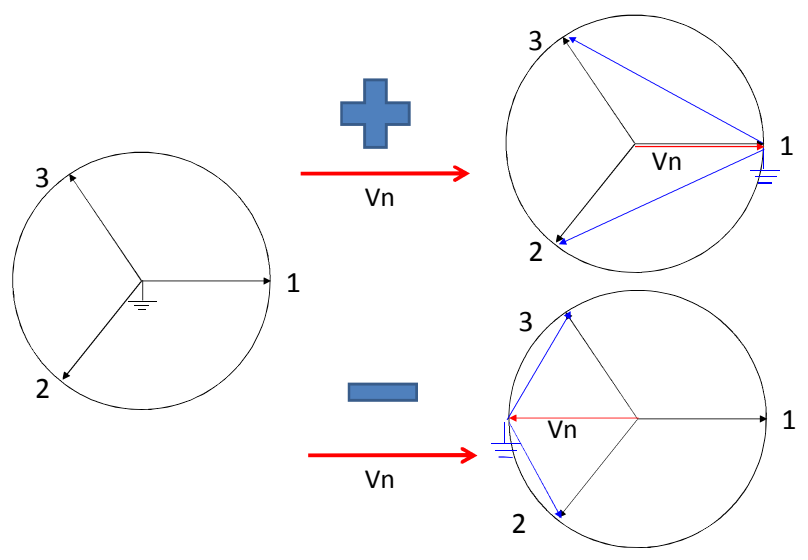

Figure 1: Any phase to earth voltage can be created by adding the appropriate zero-sequence voltage

The aim in developing the active earthing system is to incorporate new features, related to system maintenance and operation.

For maintenance purposes, it will improve weak insulation detection, while the operation will take advantage of a better detection of high impedance faults. A particular improvement in this area will be the identification of dangerous situations such as a direct contact of live conductors to earth. The enhanced earth-fault location will be useful for both purposes.

As a consequence, some faults will be prevented from appearing in the distribution network and those that happen will be promptly detected and located, thus improving continuity of supply and power quality.

This active system will behave as a low earth-fault current earthing network, but with additional features which dramatically improve the weaknesses of isolated or compensated networks.

Regarding material stress due to overvoltages, the system isolation can be periodically checked so the duration of the overvoltage will be limited, leading to a reduced impact on material.
The active earthing system comprises an innovative protection system for the enhancement of earth-fault detection, faulted phase and feeder identification and location.

\section{Active Earthing system description}

This new active earthing system for the MV distribution network, as shown in Figure 2, bases its operation on power electronics and combines them with a protection and control system, which obtains the necessary measurements to detect and locate the fault.

Zero sequence current flow and zero sequence voltages are determined by the network configuration. For a given configuration, if a current controlled in magnitude, phase and frequency is injected into the network, it is possible to determine network parameters by measuring currents and voltages. Depending on the network parameter to be measured, the injected current is adapted, using, for instance, different frequencies for capacitance or inductance measurements. This can be done both in normal operation and in a faulty network operation.

The active earthing system operates as follows:

- It is able to perform network zero sequence impedance calculations by means of the injection of fundamental and non-fundamental (other than $50-60 \mathrm{~Hz}$ ) frequency currents, as shown in (3). This calculation is used, during normal operating conditions of the network, for the calculations for the detection and location of the fault by the earthing system.

$$
\begin{aligned}
& X_{C}^{f}=\frac{V_{N}^{f}}{I_{N}^{f}} \cdot \sin \left(\Phi_{V_{N}^{f}}-\Phi_{I_{N}^{f}}\right) \\
& X_{C}^{f 0}=\frac{f}{f 0} \cdot\left(X_{C}^{f}-X_{\text {Earthing }}^{f 0} \cdot \frac{f}{f 0}\right)
\end{aligned}
$$

- It is also able to provoke a controlled neutral voltage, not only in module and in phase, but also in time, so the voltage of each phase gets the desired voltage.

During a phase-to-earth fault in a feeder, the active earthing system performs:

- Fault location, both for transient and permanent faults, by means of an innovative and enhanced protection system and fault locator based in measurements of different frequencies generated by this new system.

$$
\begin{aligned}
& \overline{Z_{0 L}^{f 0}}=R_{0 L}+X_{0 L}^{f} \cdot \frac{f}{f 0} \\
& \text { Distance_to_fault }=\frac{\overline{Z_{0 L}^{f 0}}}{\overline{Z_{\text {kmline }}}}
\end{aligned}
$$

- Fault extinction, injecting zero-sequence power frequency current to cancel the voltage of the faulted phase applying the voltage required by solving (2), 
extinguishing transient faults and, as a result, avoiding the tripping of the feeder breaker.

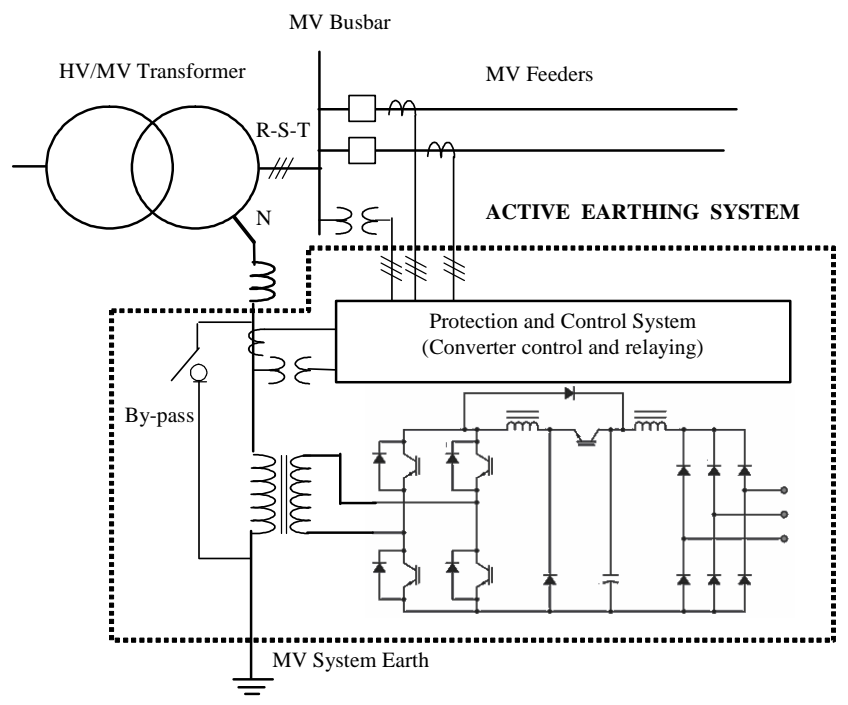

Figure 2 . Active earthing system scheme

- The reduction of faulty phase-to-earth voltage, in case of weak insulation points or partially damaged underground cables, to keep the line in service until the problem is fixed, applying the voltage required by solving (2) for the desired line voltage.

In addition, network insulation testing, for condition based maintenance purposes:

- Controlled voltage changes are used in combination with detection and location features of insulation failures, allowing the condition based maintenance of MV feeders.

- On-line network component monitoring and diagnostic can be done in combination with partial discharge detection devices installed in substations or portable detectors can be used for weak insulation points detection.

\section{Scale model system}

The objective of the scale model system is guarantying the suitable response of this system and the correct operation of the network during field tests. The influence of the levels of load currents and voltages on the validation is small, since these do not affect the zero-sequence components of actual networks. Therefore, a low power model is a useful tool to validate the system, in conditions more realistic than simulations.

Thus, the model used comprises the active earthing system and the distribution network. This model allows testing the operation principles and the correct operation of the complete system. By means of the current injection of frequencies not existing in the network, measurements and calculations accuracies are checked. On the other hand, injections at network frequency are done in order to check how fault currents are cancelled and how controlled overvoltage can be provoked.

Nevertheless, field tests are necessary to determine the behaviour of the system in a real network, given that in this model discrete components (resistors, inductors and capacitors) are used to simulate the distributed parameters of overhead lines and cables of a real network.

\section{Description of the system}

Network model is constituted by a transformer and three feeders divided into sections and different types of loads. Several network configurations can be tested modifying the type and number of sections of these lines. Besides, it is possible to simulate earth faults for each line section with resistive fault impedances and/or defective MOV surge arresters. Power electronics and the protection system model is a precise reproduction of the real one with lower rated power. Finally, feeders and earthing currents and busbar voltages are monitored to analyse the response of the whole system.

Figure 3, shows the distribution network simulator, which its diagrams in the front, what elements of the network are modelled. In the diagram above the transformer of the substation, earthed through an inductor and the connection of the active system by a transformer is included. In the one below, three lines composed by different sections, loads and resistances to generate the fault where it is required.

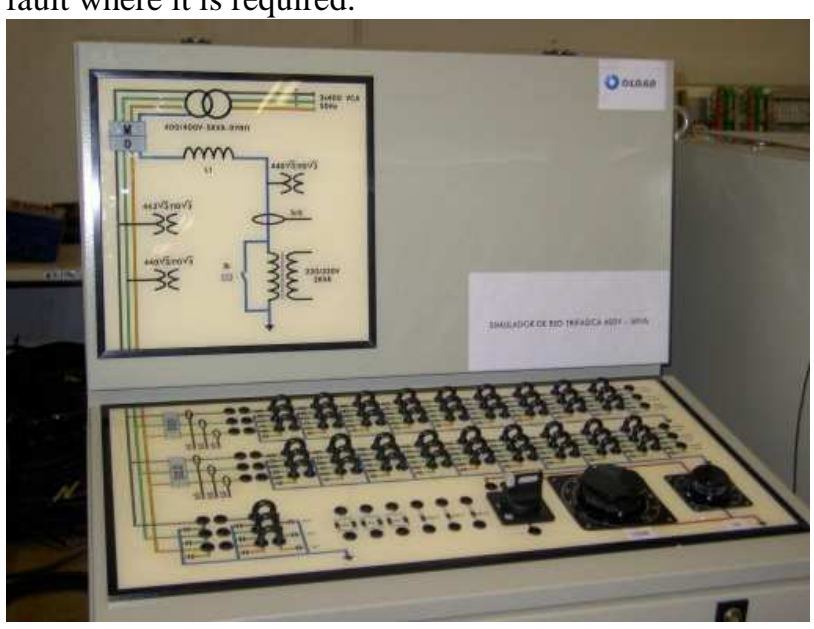

Figure 3: Distribution network simulator

Laboratory testing results

Laboratory tests, described in [2], with the scale model include:

- Measurements accuracy

- Controlled phase overvoltage

- Detection and location of weak insulation points

- Fault location

- Fault extinction

In addition, testing of new protection concepts has been carried out.

The system can work in two modes, normal (exploitation) mode and maintenance mode.

In the normal mode, faults can be easily detected by the existence of zero sequence voltage.

However, during the maintenance mode a zero sequence voltage is always present, since it is injected by the active network in order to check the isolation, as can be seen in figure 4 . 


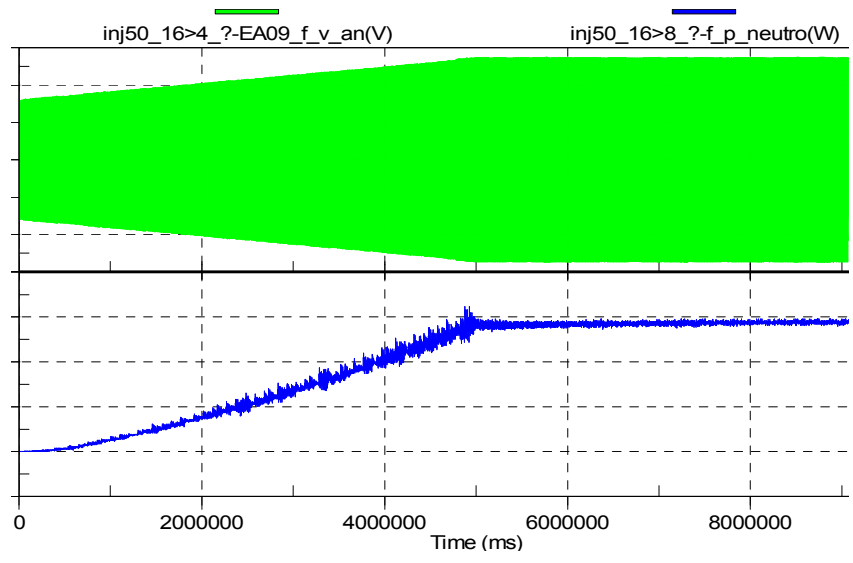

Figure 4: Phase to earth voltage increase vs. active power supplied by the earthing system in maintenance mode

For this reason, a new detection system has been implemented, in which the active power injected by the active earthing in the zero sequence circuit is examined. Figure 5 compares the active power in a healthy feeder (blue) with the active power of the same feeder having a defective surge arrester. The abnormal active power increase is detected and limited by the earthing system, being able to detect the lack of isolation before the defective element is seriously damaged.

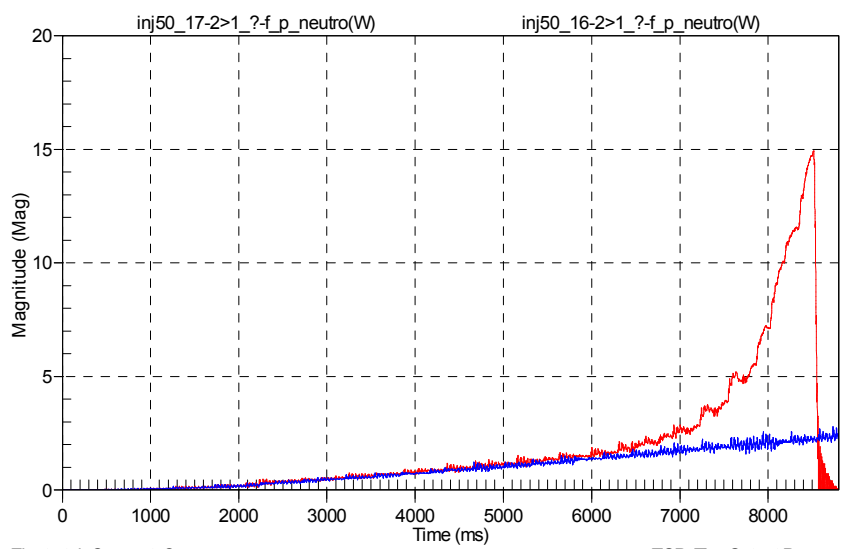

Figure 5: Active power supplied in healthy and low isolation conditions

Another crucial aspect checked in laboratory is the detection of network topology changes, since the system must be aware of the system capacitance. In addition to a periodical checking, the active earthing detects high frequency transients, representative of switching events in the network.

\section{Field installation description}

One of the most important issues when installing a new device, is to assure the correct response of the protection system in the new situation while maintaining highest safety standards (low level of touch and step voltages) for personal and equipment protection.

It has to be taken into account that the network is reconfigured as high impedance earthed while in operation without faults, and it performs as resonant earthed when injecting for fault extinction.

Regarding protection system, when the active earthing system is installed in a substation solidly or low impedance earthed, as it is the case in Gernika, no changes are expected to be required in relation with the overcurrent protections system. This protection diagram will not operate until the active earthing system finishes extinguishing the fault.

For isolated or high impedance earthed networks, minor changes might be needed in the protection system in order to maintain the protection operation diagram. The modification must delay the activation of the overcurrent protection about 100 milliseconds, the time needed for the active earthing system to control de current of the neutral. Besides, zero sequence overvoltage protection will be inhibited during the process of the extinction of the fault.

One of the most important issues when changing the earthing system is to ensure safety of persons and goods. This is accomplished by the correct operation of the protection system and the level of touch and step voltages in the new situation. It has to be borne in mind that the active network is earthed through high impedance while in normal service and that it behaves like a resonant earthed one when injecting for the extinction of the fault.

On the other hand, with this novel earthing system, the isolation level requirements are similar to those of the resonant networks. Isolation condition has been always an issue when changing an existing MV network from low to high impedance earthing. Typically, during the first months, or even years, the higher stress for isolation of high impedance earthing makes insufficient isolation appear in elements, with the consequent effect on power quality.

The traditional solution to this problem is a revision campaign of the whole MV system, previous to the earthing change, where any doubtful isolation should be replaced. However, this process is slow, costly and unreliable, since some failures (i.e. defective cable joints) cannot be easily detected. The active earthing system facilitates this process, since it allows checking the network isolation during the commissioning process. A phase by phase checking process can be applied to each feeder, locating defective points either by means of the high impedance fault detection included in the system protection or using external detection based in partial discharges.

The use of active earthing system implies using a protection system able to work with the signals injected by the converter. This can be done at different levels:

- With feeder protection relays with adapted features for earth fault detection (normal characteristics for the rest)

- With a protection relay dealing with the earth fault detection of several feeders, keeping the existing relays for the rest of functions. 


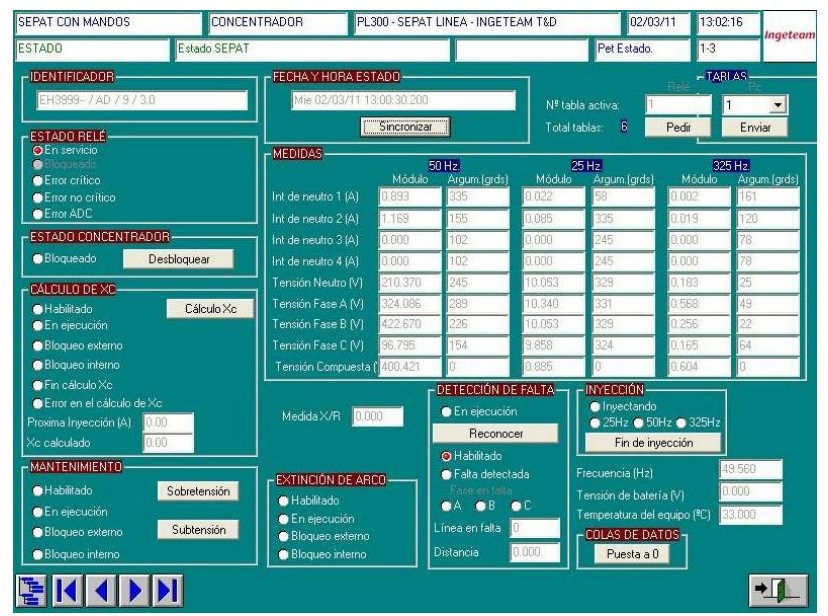

Figure 6: Protection relay control and monitoring HMI

- Maintaining the existing relays and installing a by-pass breaker, which will close in case of permanent fault, allowing the existing protection system to work normally. In this case, part of the protection functions (i.e. fault location) would not be available.

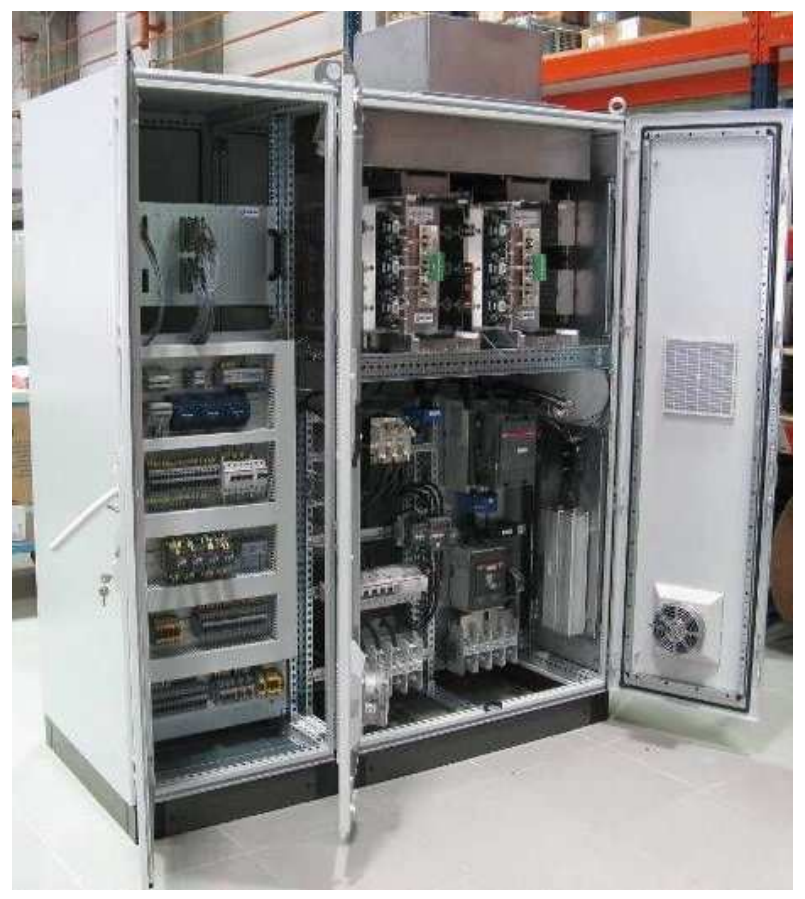

Figure 5: Active earthing converter

\section{Conclusion}

The novel earthing system presented allows network operation and maintenance and continuity of supply of the MV distribution network in which it is installed by overcoming the limitations of the traditional earthing systems used currently in distribution network MV substations due to the capability of controlling the neutral current and voltage of the network. It is based on the combination of a multi-frequency power converter and a protection and control system.

Mentioned improvements, not together achievable by means of conventional earthing systems, are summarised as follows:

- Transient earth-fault extinction

- Fault detection

- Faulted feeder identification and fault location

- Network condition oriented maintenance

- In case of a weak insulation points or partially damaged underground cables, the new system will give the chance to reduce faulted phase-to-earth voltage, to keep the line energised while the problem is fixed.

Laboratory tests results allow us to conclude the following:

- The network is protected having low step and touch voltages

- The measurement at other frequencies than $50-60 \mathrm{~Hz}$ improves accuracy in fault detection and location

- Fault current can be cancelled almost completely, to make its extinction possible

- The active earthing system allows operating networks with permanent faults in a safe way

- Response times allow carrying out the location and extinction of transient faults

All the functionalities of this patented system, based on power electronics technology, lead to an improved network performance for earth-faults, providing a real possibility to carry out condition oriented maintenance.

\section{References}

[1] F.J. Pazos, 2009, "Active grounding system for MV networks by means of power electronics", CIRED 2009, paper 0158

[2] A. Amezua, 2011 "Experimental validation results of the active grounding system for MV networks", CIRED 2011, paper 560 